\title{
Vasoproliferative tumours of the retina
}

Heinrich Heimann, Norbert Bornfeld, Oliver Vij, Sarah E Coupland, Nikolaos E Bechrakis, Ulrich Kellner, Michael H Foerster

\begin{abstract}
Background-Vasoproliferative tumours of the retina (VPTR) are benign tumours of unknown origin, occurring mostly in otherwise healthy patients. VPTR may be associated with other chorioretinal diseases, such as uveitis. The tumours, which histologically represent reactive gliovascular proliferations, are characterised by a pink to yellow appearance on funduscopy and are accompanied by exudative and haemorrhagic changes of the retina. Methods-22 cases of VPTR in 21 patients were examined with a follow up period between 1 month and 6 years. Ophthalmological changes associated with VPTR were intraretinal and subretinal exudations $(n=18)$, exudative detachments of the surrounding sensory retina $(n=13)$, intraretinal and subretinal haemorrhages $(n=10)$, exudative changes within the macula $(n=10)$, hyperpigmentation of the retinal pigment epithelium at the border of the exudative retinal changes $(n=9)$, and vitreous haemorrhages $(n=4)$. Tumour biopsy was performed in two cases. Treatment consisted of plaque radiotherapy $(n=14)$, plaque radiotherapy and cryotherapy (two), cryotherapy only (two), observation (three), and enucleation in one case of a blind and painful eye.
\end{abstract}

Department of Ophthalmology, University Hospital Benjamin Franklin, Free University, Berlin, Germany H Heimann

N E Bechrakis

U Kellner

M H Foerster

Department of Ophthalmology, University Hospital, Essen, Germany $\mathrm{N}$ Bornfeld

$\mathrm{O}$ Vij

Department of Pathology, University Hospital Benjamin Franklin, Free

University, Berlin, Germany

S E Coupland

Correspondence to: Dr Heinrich Heimann, Augenklinik, University Hospital Benjamin Franklin, Hindenburgdamm 30 D-12200 Berlin, Germany heimann@ukbf.fu-berlin.de

Accepted for publication 16 May 2000
Results-Regression of the tumour and the associated exudative changes could be observed in all treated cases. Visual acuity at last follow up improved two lines or more in two cases, remained within two lines of the initial visual acuity in 15 cases, and worsened in the remaining five. Histopathological examination of the biopsy specimens and the tumour of the enucleated eye showed massive capillary proliferation with perivascular spindleshaped glial cells of retinal origin.

Conclusion-The correct diagnosis of VPTR is of importance as these lesions may lead to visual loss. Further, VPTR must be differentiated from angiomas associated with von Hippel-Lindau disease as well as from ocular and systemic malignancies. Regression of tumour thickness and associated retinal changes can be achieved with brachytherapy or cryotherapy.

(Br f Ophthalmol 2000;84:1162-1169)

Vasoproliferative tumours of the retina (VPTR) are benign lesions of unknown origin, occurring mostly in otherwise healthy patients between 40 and 60 years of age. ${ }^{1}$ These highly vascularised tumours are characterised by a pink to yellow colour on funduscopy and are associated with intraretinal haemorrhages, intraretinal or subretinal exudates, and hyperpigmentations of the retinal pigment epithelium.

The pathogenesis of these lesions has yet to be clarified. Some reports of VPTR in the literature have documented their association with other diseases affecting the retina or choroid, such as uveitis, retinochoroiditis, sickle cell retinopathy, retinitis pigmentosa, retinopathy of prematurity, or long standing retinal detachment. ${ }^{2-9}$ In the majority of cases, however, such an association was not observed. ${ }^{1}{ }^{10}$

The correct diagnosis of VPTR is of clinical importance as these lesions may lead to visual loss that can be prevented with appropriate treatment. Further, these tumours must be differentiated from angiomas associated with von Hippel-Lindau disease (VHLD), as well as from ocular and systemic malignancies with retinal involvement.

Patients and methods

Between April 1993 and July 1999, 22 vasoproliferative tumours of the retina were diagnosed in 21 patients in the departments of ophthalmology at the University Hospital Benjamin Franklin in Berlin and at the University Hospital in Essen, Germany. All patients received a thorough ophthalmological examination, including funduscopy with photographic documentation, fluorescein angiography, A and B scan ultrasonography. Further, an extensive systemic examination including magnetic resonance imaging (MRI) of the brain was performed and family histories taken to exclude diseases, such as Von-Hippel Lindau, associated with retinal vascular tumours. Serological screening for mutations of the VHLD gene was not routinely performed.

Tumour biopsy was required to confirm the diagnosis of VPTR in two cases; enucleation was necessary in one patient due to a blind and painful eye. The biopsy specimens were fixed in $4 \%$ formalin, embedded in paraffin, and serially sectioned. The enucleated globe was similarly placed in formalin and, following fixation, opened horizontally after transillumination to include the tumour in the central pupil optic nerve/macular block. Conventional histological stains included haematoxylin and eosin, periodic acid Schiff, and elastin. Additional slides were stained for immunohistochemical studies using standard techniques ${ }^{11}$ and several monoclonal antibodies, including glial fibrillary acidic protein (GFAP, Dako), JC70a (CD31, Dako), and MIB-1 (Ki-67, Dako), reactive in paraffin sections. 


\section{Results}

Seven female and 14 male patients were examined; the age at presentation ranged from 15.1 to 70.1 years (mean 46.7 years). A history of Von-Hippel Lindau disease could be excluded in all patients.

Seventeen patients had presented to their ophthalmologists with symptoms of visual loss and/or "floaters" or photopsia. One patient presented with a blind and painful eye due to neovascular glaucoma and a history of bilateral recurrent uveitis. In four cases, no symptoms were reported and the tumours were observed during routine examination, one of them in the fellow eye of a patient already being treated for VPTR for 1 year. Visual acuity on first examination in our institutions was between no light perception and 6/6 (median 6/18).

Previous eye diseases in the eyes affected by VPTR included chronic recurrent uveitis (five patients, bilateral in two patients), retinitis pigmentosa (two), history of retinal detachment surgery (two), toxoplasmosis retinochoroiditis (one), and retinal break (one). In the remaining 10 patients, the ophthalmological history was unremarkable. Medical history included hypertension (three patients), chronic heart failure (one), and carcinoma of the breast treated with mastectomy (one).

On funduscopy, the tumours appeared as solid pink to peach coloured masses within the retinal plane; all but two tumours were located at the equatorial region of the lower retinal periphery (Figs 1 and 2). Two tumours were observed central to the equator within the upper nasal quadrant. Additional clinical changes associated with the tumours were intraretinal and subretinal exudations (18 of 22 cases), exudative detachments of the surrounding sensory retina (13/22), intraretinal and subretinal haemorrhages (10/22), hyperpigmentation of the retinal pigment epithelium at the border of the exudative retinal changes $(9 / 22)$, cells in the anterior chamber $(4 / 22)$, and vitreous haemorrhages (4/22). Exudative macular changes were present in 10 patients.

Fluorescein angiography of sufficient quality could be obtained in 10 cases. A connection between retinal feeder vessels and a rich capillary network within the tumours (Fig 1C, D, and $2 \mathrm{H}$ ) could be demonstrated in all of these cases. In contrast with typical findings in retinal angiomas associated with VHL, the retinal feeder vessels were not or only mildly dilated. Leakage of dye from tumour vessels was seen during the arteriovenous passage and in the later frames of fluorescein angiography in all examined tumours. Telangiectactic dilations of tumour vessels were noted in seven cases (Fig 1A).

B-scan ultrasonography demonstrated solid tumours in all cases. Low, medium, and high internal reflectivities could be measured with A-scan ultrasonography in different tumours with no distinct pattern of internal reflectivity applicable to all tumours. Tumour thickness varied between 1.0 and $5.6 \mathrm{~mm}$ (mean 2.8 $\mathrm{mm})$.

In three cases, the diagnosis of VPTR could not be made based on the clinical examination alone. In one patient, the diagnosis was made upon histological examination following enucleation of a blind and painful eye with an intraocular mass (Fig 1G). Tumour biopsies following pars plana vitrectomy were performed in the two remaining patients (one patient with a history of carcinoma of the breast to rule out retinal/choroidal metastasis, one patient with a large tumour of $5.6 \mathrm{~mm}$ thickness and marked accompanying exudations (Fig 2A-F)). The consistent finding in the biopsy cases, as well as in the tumour of the enucleated eye, was a proliferation of slender spindle-shaped cells with eosinophilic cytoplasm, small uniform nuclei and no signs of pleomorphism (Figs 1H-J and 2E). The spindle-shaped cells stained positively for GFAP, therefore, supporting the glial origin of the cells. The glial cell proliferation was imposed over a fine capillary network, whose endothelium stained positively for CD31. Another dominant feature of the lesions was the presence of dilated blood vessels, characterised by marked hyalinisation of the vessel walls. Some of the vessels were occluded; however, others remained patent. Necrosis, haemorrhage, and calcification were absent. The growth fraction of the spindle cells, as determined using MIB-1 antibody, was low (maximum 5\%). Similarly, the growth fraction of the endothelium of non-hyalinised blood vessels varied between 1 and 3\% (not shown).

Treatment of the patients with VPTR consisted of "close observation" in one case of an amblyopic eye with a tumour (largest dimension $3.9 \mathrm{~mm}$ ) and associated macular changes, as an improvement of visual function was not thought to be achievable through therapeutic interventions. Two patients refused the proposed brachytherapy and cryotherapy. In these cases, VPTR were previously diagnosed as "retinal teleangiectasis" and "retinal granuloma"; they were followed for several months by other ophthalmologists before they were seen in our departments. As mentioned above, enucleation was performed in one case as the eye was blind and painful. In one case with a dense vitreous haemorrhage, pars plana vitrectomy was performed before visualisation and treatment of the tumour. Cryotherapy alone was applied to two small tumours with a thickness of less than $2 \mathrm{~mm}$. In another two cases, cryotherapy was tried as the first intervention in tumours with a thickness of more than $2 \mathrm{~mm}$; however, as the entire lesions could not be frozen in one session, additional brachytherapy was applied in these cases. The remaining 14 cases were treated with $\beta$ ray brachytherapy using 106-ruthenium/106rhodium plaques (Bebig, Germany). In these cases, a scleral contact dose of 500 Gy was applied, although in two larger tumours, scleral contact doses of 1000 Gy and 870 Gy were used. The mean scleral contact dose was 677 Gy, resulting in a mean apex dose of 81 Gy.

The follow up period varied between 1 month and 6 years (mean 26 months). During the follow up period, the tumour in the amblyopic eye which was not treated actively did not show any signs of further growth or progressive 

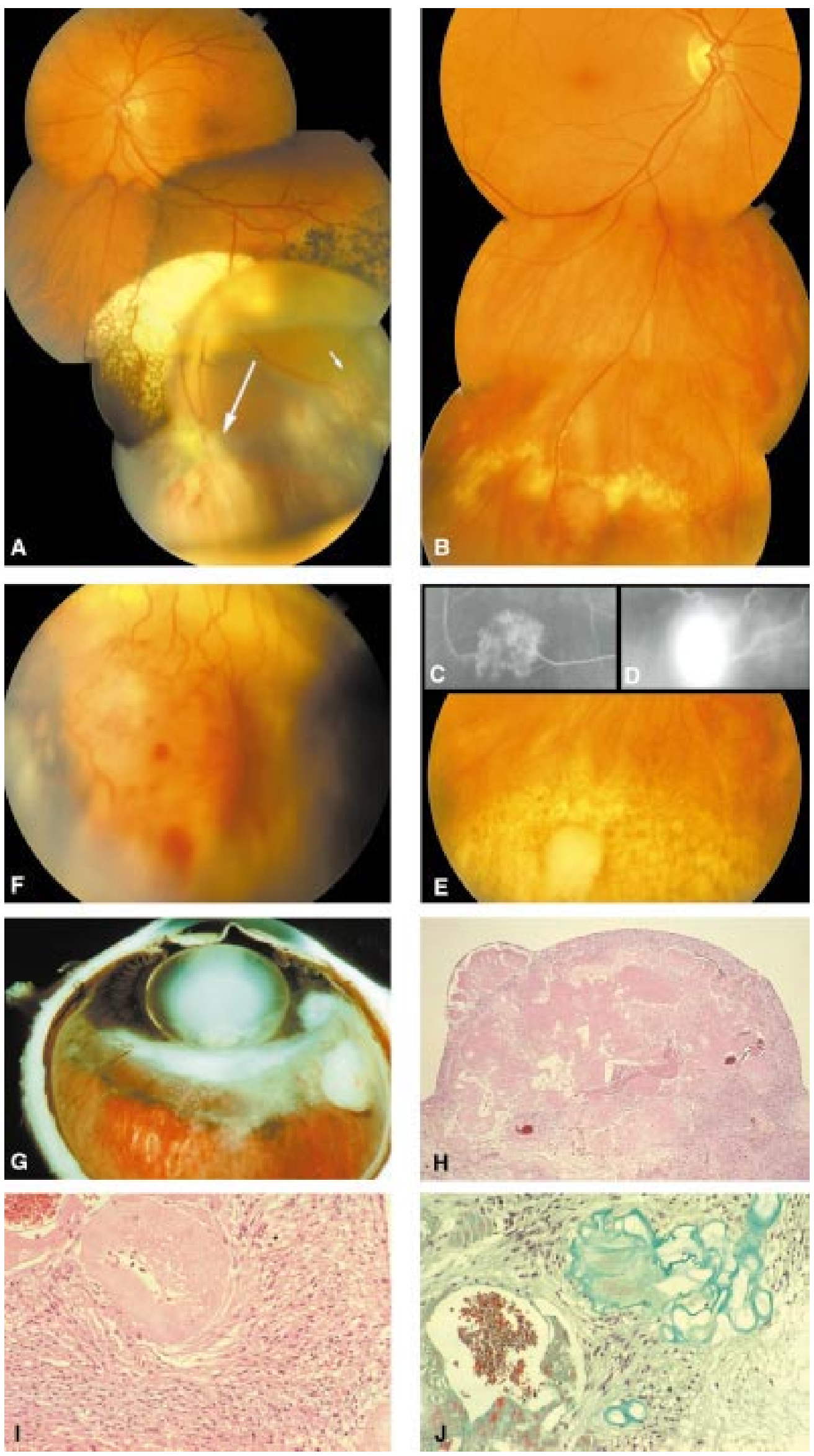

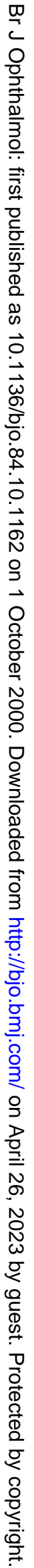



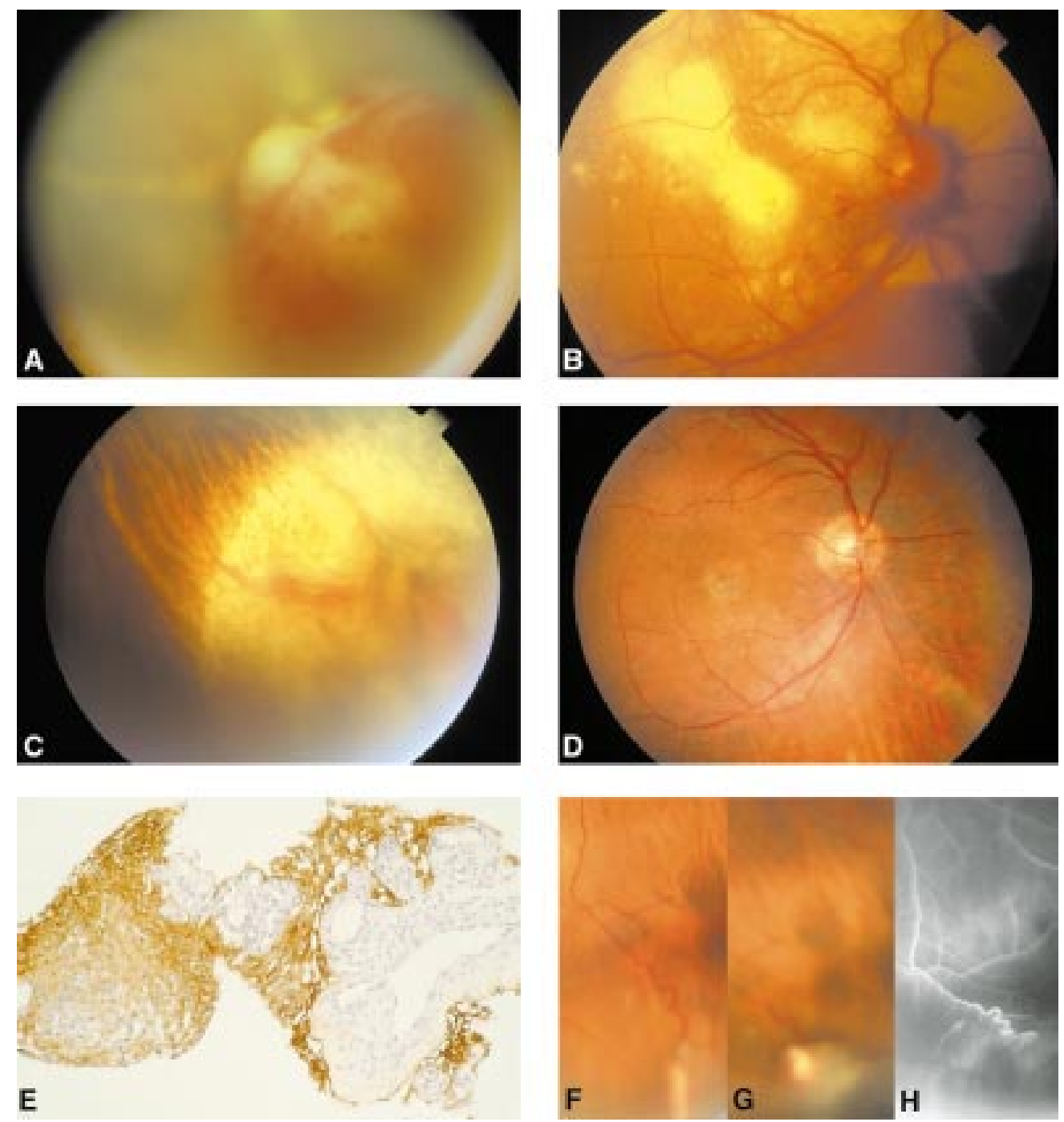

Figure 2 (A) VPTR of the lower nasal periphery of the right eye in a 31 year old male patient. Although the patient complained of visual loss and floaters for more than 6 months, diagnosis was delayed because no fundus examination had been performed by his ophthalmologist during this period. The tumour was biopsied following pars plana vitrectomy. (B) Massive intraretinal and subretinal exudations extending to the central retina in the same patient as $(A)$, accompanied by retinal neovascularisations on the optic disc. (C) Same patient as in (A). Chorioretinal scar and complete regression of VPTR after 106-ruthenium brachytherapy. (D) Same patient as in (B). Complete regression of the central retinal exudates 1 year after 106-ruthenium brachytherapy. Note the residual changes of the RPE within the central macula. No significant gain in visual acuity could be noted despite regression of the exudative changes. (E) The biopsy specimen obtained from the gain in visual acuity could be noted despite regression of the exudative changes. (E) The biopsy specimen obtained from the
tumour displayed in Figure 1 stained for glial fibrillary acid protein (GFAP, $\times 40$ objective), demonstrating that the spindle cell population within the retinal tumours are of glial cell origin. The endothelium of the blood vessels is negative for this marker. (F) Left eye of the same patient as in (A). Initially, no fundus abnormalities could be noted in his fellow eye. After 1 year of follow up, a small VPTR could be seen in the lower retinal periphery of his fellow left eye. (G) Same tumour as in $(F)$ demonstrating tumour growth after 4 weeks of follow up. The tumour was then treated with cryotherapy (picture taken with scleral indentation). (H) Fluorescein angiography of the same tumour as in (G) demonstrating the connections between the retinal vasculature and the tumour vessels.

exudative changes of the retina; hence, no further treatment was performed in this case. In one patient who refused cryotherapy, slow tumour growth from $1.6-2.1 \mathrm{~mm}$ could be observed over a period of 28 months. In another patient who refused therapy, no progression of a tumour with an initial thickness of $1.8 \mathrm{~mm}$ could be seen over a period of 4 years. All treated tumours showed a regression in thickness and associated exudative changes. Visual acuity at last follow up was improved two lines or more in two cases,

Figure 1 (A) VPTR in the lower retinal periphery in a 31 year old male patient who presented with floaters and loss of vision. The tumour (long arrow) is accompanied by an exudative retinal detachment, intraretinal accumulations of lipoid exudates, telangiectactic alterations of the retinal vessels (small arrow), and marked hyperpigmentations of the retinal pigment epithelium in the retina adjacent to the exudative detachment. (B) Symptomless VPTR in the lower retinal periphery in a 65 year old female patient. (C) Mid-phase fluorescein angiography of the tumour shown in (B) demonstrating the connection of tumour vessels with non-dilated retinal vessels. (D) Late phase fuorescein angiography of the tumour shown in (B) demonstrating preretinal leakage of dye on the tumour surface. (E) VPTR as shown in $(B, C) 6$ months after brachytherapy with 106-ruthenium. Regression of tumour thickness and exudative retinal changes. Chorioretinal scarring within the area that was covered by the 106-ruthenium plaque. (F) VPTR in the lower retinal periphery of a 15 year old boy with a history of toxoplasmosis retinochoroiditis. (G) Enucleation specimen with VPTR of the retinal periphery in a 27 year old male patient who presented with a blind and painful eye as a result of neovascular glaucoma. (H) Low power magnification of the retinal tumour demonstrating that it consists of a proliferation of glial cells and small blood vessels embedded in a hyalinised stromal matrix (haematoxylin and eosin stain, $\times 10$ objective). (I) High power magnification of a hyalinised blood vessel surrounded by glial cells (haematoxylin and eosin stain, $\times 40$ objective). (7) High power magnification of a blood vessel filled with erythrocytes and surrounded by glial cells and hyalinised stroma (Gomori stain, $\times 40$ objective). (A and $B$ were assembled using digitised fundus photographs and Adobe Photoshop software). 
remained within two lines of the initial visual acuity in 15 cases (including the three cases in which no treatment was employed) and worsened in the remaining five (corneal endothelial decompensation in one case and presumed macular dysfunction secondary to long standing exudative changes of the macula in four cases). Neither progression or recurrence of the treated tumours could be detected during the follow up period. Further, no typical complications of brachytherapy, such as the development of radiation induced cataract or retinopathy, were observed in the patients treated with brachytherapy.

\section{Discussion}

Vasoproliferative tumours of the ocular fundus have only recently been defined as a distinct entity in the differential diagnosis of intraocular tumours. ${ }^{1891213}$ Essentially, these benign lesions represent reactive gliovascular proliferations, with varying degrees of both gliosis and of vascular proliferation, as demonstrated in the histopathological specimens in our series (Fig 1H-J, Fig 2E) and by others. ${ }^{1814}$ Whether the gliosis or the vascular component is the initiating or driving element in this process remains a point of conjecture and requires further investigation.

Possibly because of the varying extent of gliosis and vascular proliferation, considerable confusion exists concerning the nomenclature of VPTR in the literature. In those lesions where the vascular proliferation dominated the clinical and histological picture they have previously been termed "retinal angiomas in the aged", 15 "angioma-like lesion", " "angiomalike mass", 2 "neovascular fundus abnormalities", "presumed acquired retinal hemangiomas", ${ }^{16}$ "peripheral retinal teleangiectasis", "retinal angiomatous mass", "peripheral uveal neovascularisation, ${ }^{17}$ "haemangioma-like masses of the retina", ${ }^{18}$ or "aquired retinal angiomas". ${ }^{19}$ Further, the variation in the nomenclature and definition of VPTR could be partially owing to the fact that the tumours might be detected at different stages, ranging from small, asymptomatic fibrovascular buds (Fig 2F) or perhaps teleangiectasis to large tumorous lesions with massive exudative changes (Fig 2A).

On the other hand, where the glial component of these lesions prevailed on histological examination, it may be possible that at least some cases of VPTR have previously been categorised into the group of benign glial tumours of the retina-for example, "glial nodules", ${ }^{20}$ "(massive) retinal gliosis", ${ }^{21-24}$ or "retinal astrocytoma" not associated with tuberous sclerosis or neurofibromatosis. ${ }^{25-27}$ Indeed, the histological picture of "localised retinal gliosis" 24 is very similar to that of VPTR observed in the current study.

Although there is no single characteristic which is pathognomic for VPTR, the typical appearance on funduscopy is that of a peachcoloured, highly vascularised, solitary mass within the plane of the sensory retina (Figs 1 and 2). As seen in nine out of 10 cases in our series, the tumours are usually located in the pre-equatorial and equatorial regions of the lower retinal quadrants, particularly at the 5-7 o'clock positions. Infrequently, these tumours are seen in the upper retinal quadrants or posterior to the equator. ${ }^{11016}{ }^{18}$ Multiple tumours or bilateral involvement, as seen in one case of our series (Fig 2), is rare.

The most common retinal alterations associated with VPTR were the accumulation of hard exudates in the retina surrounding the tumour, as seen in 18 of 22 eyes of our series (Figs 1A and $2 \mathrm{~B}$ ). These changes are secondary to an increased permeability of tumour vessels compared with normal retinal capillaries as demonstrated by fluorescein angiography. They can lead to an exudative detachment of the sensory retina, potentially involving the macula. Cystoid macular oedema without macular detachment can also occur. Most of the symptoms that are reported can be attributed to exudative macular or retinal changes-for example, blurred vision and visual field loss.

Subretinal, intraretinal, and preretinal haemorrhages may also be observed in association with VPTR; these are presumed to be due to a leakage from or rupture of tumour vessels, or to alterations of the retinal vessels adjacent to the tumour. The haemorrhages can vary in severity from minor punctate intraretinal bleedings to severe vitreous haemorrhages, causing a significant loss in visual acuity. ${ }^{15} 101618$ In the current series, moderate to severe vitreous haemorrhages were seen in four eyes, requiring vitrectomy in one case.

Hyperpigmentation of the retina adjacent to the vascular retinal tumours (Fig 1A) are a further clinical characteristic of VPTR. These were observed in nine of our 22 cases and have been described by other authors. ${ }^{116}$ It has been proposed that they resemble disturbances of the retinal pigment epithelium (RPE) secondary to exudative retinal detachment. ${ }^{16}$ However, in some of our cases, the degree of hyperpigmentation exceeded that of RPE disturbances normally seen secondary to exudative detachments. Further, hyperpigmentation was also observed in retinal tissue in which there was no evidence of exudative detachment (Fig 1A). It has been proposed that this hyperpigmentation is part of the disease process, representing "an expression of vascularisation of pigment epithelial proliferation or reactive gliosis". ${ }^{1}$

There appears to be an association between VPTR and other ocular diseases, in particular, uveitis. ${ }^{8}$ The vasoproliferative tumours could possibly be a reactive process to factors released-for example, secondary to the breakdown of the blood-retinal barrier in uveitis, which may lead to an uncontrolled proliferation of fibrous tissue and angiogenesis in the retina. Although this association has been described, half of the patients in our series, similar to the majority of patients described in the literature, had no history or signs of uveitis or other retinochoroidal diseases. In these patients, an idiopathic nature of VPTR must be presumed, particularly in one case from our series (Fig 2F-H), and in three patients reported in another series, ${ }^{18}$ who all had 
normal fundus appearances before the occurrence of the tumours. Furthermore, in some cases in the literature defined as "VPTR secondary to uveitis", the intraocular inflammation and uveitis itself might also be interpreted as a reactive or "spillover" phenomena secondary to the tumorous lesion with leakage of inflammatory cells from tumour vessels into the vitreous cavity. The pathogenesis of VPTR is, therefore, at present unclear; it is likely that many different disease processes can lead to the initiation of a proliferation of retinal glial cells and blood vessels that clinically appear as VPTR.

The major differential diagnoses of VPTR are other vascular or tumorous lesions of ocular fundus, including capillary haemangiomas associated with VHLD; Coats' disease; peripheral exudative haemorrhagic chorioretinopathy (PEHC); and malignancies that potentially may involve the retina-for example, retinal/ choroidal metastasis or choroidal melanoma with secondary retinal infiltration. These different entities, and their differentiation from VPTR, will be briefly discussed.

VPTR and retinal angiomas associated with VHLD share common features, as they are vascular retinal tumours associated with exudative retinal changes. ${ }^{28}$ However, marked dilatation of retinal feeder vessels, even in relatively small tumours and a stellate macular exudate, are two characteristics of VHLD which cannot be observed in VPTR. In the latter, only a slight dilatation of retinal vessels is observed and retinal exudations are usually located around the tumour itself in the case of small lesions. Further, patients with VHLD often present at a younger age, have multiple angiomas, display other features of VHLD, and/or report a positive family history of the disease. ${ }^{16}$

The common features of VPTR and Coats' disease include teleangiectatic dilatations of retinal vessels, retinal exudations, and exudative retinal detachment. In fact, some of the cases, which we would classify as VPTR, have been published as "massive gliosis of the retina" 29 or "peripheral angioma" ${ }^{14}$ associated with Coats' disease. In contrast with VPTR, Coats' disease typically effects infant or juvenile males. Tumorous lesions in Coats' disease usually represent massive accumulations of lipid exudates and are typically accompanied by an advanced exudative retinal detachment. A distinct circumscribed or nodular intraocular mass lesion, as found in VPTR, is not a common feature even in very advanced cases of Coats' disease. $^{30}$

Amelanotic melanomas and metastasis of systemic malignancies are usually located within the choroid, in contrast to VPTR. However, metastases in the retina, as well as retinal infiltration from choroidal metastasis or melanomas, have been documented..$^{31-34}$ Shields et al described a case of a malignant melanoma that was drained by a large retinal vein, mimicking a retinal angioma. ${ }^{34}$ As in two of our patients, the diagnosis of VPTR by clinical examination alone might still not be elaborated with certainty, even if fluorescein angio- graphy or ultrasound are used. In our cases, the correct diagnosis was established following pars plana vitrectomy and tumour biopsy.

Another clinical entity that has to be differentiated from VPTR is PEHC, which is associated with peripheral retinal, subretinal, and vitreous haemorrhages, exudates, exudative retinal detachments, macular oedema, as well as tumorous lesions. In contrast with VPTR, the tumours are usually located in the subretinal space and show dark coloured or pigmented changes secondary to residues from subretinal haemorrhages on their surface. ${ }^{35}$ Tumorous manifestations which appear to lie within the plane of the retina, as well as non-pigmented yellow coloured lesions, however, have been described. ${ }^{35}$ Subretinal neovascularisations are only rarely found in PEHC, and, if fluorescein angiography can be performed, a connection between the retinal and tumour vessels is not usually seen in PEHC in contrast with VPTR. Further, PEHC is often bilateral, located in the temporal retinal quadrants, is often associated with macular degeneration and usually occurs in patients above the age of 60 years.

Although there is no single unique characteristic feature that definitely can distinguish VPTR from other intraocular tumours in every case, it is our opinion that VPTR is a different clinical entity that can in most cases be differentiated by the combination of the above mentioned clinical features. If an intraocular malignancy cannot be ruled out by clinical appearance and ancillary examinations alone, we are of the opinion that intraocular tumour biopsy in such patients is justified.

The natural course of VPTR appears to be variable. A cessation in or only slow disease progression has been observed in some cases. ${ }^{1013}$ On the other hand, progressions complicated by tractional retinal detachments have been described. ${ }^{9}$ In our series, definite enlargement of relatively small tumours occurred in two patients. In another two cases, in which no treatment was employed, no progression was seen over 1 and 4 years, respectively. However, these two cases were already complicated by exudative macular changes that resulted in a loss of visual acuity. In the remaining cases, the natural progression could not be evaluated as treatment was applied. As most patients presented with symptomatic lesions, and since the symptoms are usually related to increasing exudations from the vasoproliferative tumours, it is our impression that progression of the disease process and a decrease in visual acuity are likely to occur in most cases.

The indications for and modalities of treatment for VPTR have not yet been clearly defined. Most authors agree that treatment is indicated for lesions associated with complications which lead to a loss in vision. ${ }^{16}{ }^{18}$ Some authors advocate treatment at an early stage because smaller lesions are easier to treat and early treatment might save visual acuity before the occurrence of any symptoms. ${ }^{10}$ Our data from patients with long standing macular oedema, in whom an improvement of visual 
acuity despite both regression of the lesion and the exudative changes of the retina was not achieved, would support this notion. Modalities that have been employed for the treatment of VPTR include perforating diathermy, ${ }^{536}$ laser photocoagulation, ${ }^{5}$ cryotherapy, ${ }^{1} 101618$ plaque radiotherapy, ${ }^{1}$ and vitrectomy for associated tractional retinal detachment or macular pucker. ${ }^{12}$

Cryotherapy seems to be sufficient for the treatment of small tumours $(<2 \mathrm{~mm})$. Larger tumours are, however, difficult to treat with cryotherapy, as tumour thickness may hinder complete treatment in one session. ${ }^{10}$ Further, freezing of larger lesions can lead to an increase in associated exudative changes ${ }^{10}$ and, possibly, complete exudative detachment. ${ }^{18}$ Failure of treatment with cryotherapy alone has recently been described in two of five patients by Lafaut et al in VPTR associated with presumed congenital toxoplasmosis. ${ }^{9}$

In the current series, plaque radiotherapy for VPTR with a thickness of $2 \mathrm{~mm}$ or more was applied, owing to encouraging results with 106-ruthenium brachytherapy for retinal haemangiomas of comparable size and location. ${ }^{37}{ }^{38}$ The advantage of this therapy was the possibility of treating the entire lesion, including the apex of the tumour with relative ease and safety due to the peripheral location of these lesions. Further, direct tumour vascular damage, as seen with cryotherapy or photocoagulation which may subsequently lead to immediate haemorrhage or exudation, is very unusual in brachytherapy. The dosage required for sufficient treatment of VPTR using brachytherapy has not yet been determined; however, choroidal haemangiomas $^{38}$ seem to require lower dosages compared to retinal angiomas. ${ }^{37}$ In this series, a mean scleral contact dose of 677 Gy and a mean apex dose of 81 Gy were sufficient to cause regression of all radiated tumours and associated exudations. Further, a stabilisation or improvement of visual acuity was observed in most cases treated with 106-ruthenium plaque radiotherapy. The exceptions were one case with increasing corneal oedema due to endothelial decompensation in association with chronic recurrent uveitis and four cases with associated exudative changes of the macula and a low initial visual acuity, despite regression of tumour thickness and regression of the macular changes (see Fig 2A-D). This presumably was caused by permanent photoreceptor damage secondary to exudative changes of the macula.

The possible disadvantages of plaque radiotherapy include cataract formation and radiation retinopathy. ${ }^{39}$ The occurrence of these complications depends upon the location and size of the tumour and the dose of radiation applied. Neither increased cataract formation nor radiation retinopathy could be observed in this series with a mean follow up of 2.8 years. Reducing the risk of radiation retinopathy in VPTR is the relative small size of the tumours and their anterior location.

In conclusion, VPTR are benign retinal gliovascular proliferations of idiopathic and, in some cases, presumed secondary nature that are important in the differential diagnosis of tumorous lesions of the ocular fundus. Histological examination of three tumours supported the retinal origin of these lesions. Treatment is indicated for VPTR associated with retinal changes that potentially can cause loss in function. Regression of tumour thickness and associated retinal changes could be achieved with plaque radiotherapy in VPTR with a thickness of $2.0 \mathrm{~mm}$ or more and with cryotherapy in smaller tumours $(<2 \mathrm{~mm})$.

This paper was presented in part at the annual meeting of the Association for Research in Vision and Ophthalmology, Fort Lauderdale, Florida, USA, in May 1997 and at the 95th congress of the German Ophthalmologic Society, Berlin, Germany in September 1997.

None of the authors has a proprietary interest associated with the contents of this article.

1 Shields CL, Shields JA, Barrett J, et al. Vasoproliferative tumors of the ocular fundus. Classification and clinical tumors of the ocular fundus. Classification and clinical manifestations in

2 Barr CC, Rice TA, Michels RG. Angioma-like mass in a patient with retrolental fibroplasia. Am $\mathcal{F}$ Ophthalmol 1980; 89:647-50

3 Cardoso RD, Brockhurst RJ. Perforating diathermy coagulation for retinal angiomas. Arch Ophthalmol 1976;94:170215

4 Galinos SO, Smith TR, Brockhurst RJ. Angioma-like lesion in hemoglobin sickle cell disease. Ann Ophthalmol 1979;11: 1549-52.

5 Felder KS, Brockhurst RJ. Neovascular fundus abnormalities in peripheral uveitis. Arch Ophthalmol 1982;100:750-4.

6 Gottlieb F, Fammartino JJ, Stratford TP, et al. Retinal angiomatous mass. A complication of retinal detachment surgery. Retina 1984;4:152-7.

7 Medlock RD, Shields JA, Shields CL, et al. Retinal hemangioma-like lesions in eyes with retinitis pigmentosa. Retina 1990;10:274-7.

8 Smeets MH, Mooy CM, Baarsma GS, et al. Histopathology of a vasoproliferative tumor of the ocular fundus. Retina of a vasoprolifer $1998 ; 18: 470-2$.

9 Lafaut BA, Meire FM, Leys MA, et al. Vasoproliferative retinal tumors associated with peripheral chorioretinal scars in presumed congenital toxoplasmosis. Graefes Arch Clin Exp Ophthalmol 1999;237:1033-8.

10 Laqua H, Wessing A. Peripheral retinal telangiectasis in adults simulating a vascular tumor or melanoma. Ophthalmology 1983;90:1284-91.

11 Cordell JL, Falini B, Erber WN, et al. Immunoenzymatic labeling of monoclonal antibodies using immune complexes of alkaline phosphatase and monoclonal antialkaline phosphatase (APAAP complexes). 7 Histochem Cytochem 1984;32:219-29.

12 McDonald HR, Schatz H, Johnson RN, et al. Vitrectomy in eyes with peripheral retinal angioma associated with traction macular detachment. Ophthalmology 1996;103: 329-35; discussion 334-5.

13 McCabe CM, Mieler WF. Six-year follow-up of an idiopathic retinal vasoproliferative tumor. Arch Ophthalmol 1996;114:617.

14 Henkind P, Morgan G. Peripheral retinal angioma with exudative retinopathy in adults (Coats's lesion). Br f Ophthalmol 1966;50:2-11.

15 Schmitz-Valckenberg P, Meyer-Schwickerath G. Retinal angiomatosis in the aged. Ber Zusammenkunft Dtsch Ophthalmol Ges 1977;74:205-8.

16 Shields JA, Decker WL, Sanborn GE, et al. Presumed acquired retinal hemangiomas. Ophthalmology 1983;90: 1292-300.

17 Kaba F, el Baba F, Green WR. Uveal neovascularization at the ora serrata and pars plana. Ann Ophthalmol 1987;19: 85-90.

18 Campochiaro PA, Conway BP. Hemangiomalike masses of the retina. Arch Ophthalmol 1988;106:1409-13.

19 Palmer JD, Gragoudas ES. Advances in treatment of retinal angiomas. Int Ophthalmol Clin 1997;37:159-70.

20 Ganley JP, Streeten BW. Glial nodules of the inner retina. Am f Ophthalmol 1971;71:1099-103.

21 Berger B, Peyman GA, Juarez C, et al. Massive retinal gliosis simulating choroidal melanoma. Can $\mathcal{F}$ Ophthalmol 1979;14:285-90.

22 Bovino JA, Marcus DF, Nelsen PT. Fluorescein angiography in massive retinal gliosis. Am $\mathcal{F}$ Ophthalmol 1987;103:593.

23 Rodrigues MM, Bardenstein D, Wiggert B, et al. Retinitis pigmentosa with segmental massive retinal gliosis. An immunohistochemical, biochemical, and ultrastructural study. Ophthalmology 1987;94:180-6.

24 Sahel JA, Frederick AR Jr, Pesavento R, et al. Idiopathic retinal gliosis mimicking a choroidal melanoma. Retina 1988;8:282-7.

25 Bloom SM, Mahl CF. Photocoagulation for serous detachment of the macula secondary to retinal astrocytoma. Retina 1991;11:416-22. 
26 Jakobiec FA, Brodie SE, Haik B, et al. Giant cell astrocytoma of the retina. A tumor of possible Mueller cell astrocytoma of the retina. A tumor of possib
origin. Ophthalmology 1983;90:1565-76.

27 Ramsay RC, Kinyoun JL, Hill CW, et al. Retinal Ramsay RC, Kinyoun JL, Hill CW, et
astrocytoma. Am f Ophthalmol 1979;88:32-6.

28 Welch RB, discussion in Shields JA, et al. Presumed acquired retinal hemangiomas. Ophthalmology 1983;90:1300.

29 Green WR. Bilateral Coats' disease. Massive gliosis of the retina. Arch Ophthalmol 1967;77:378-83.

30 Haik BG. Advanced Coats' disease. Trans Am Ophthalmol Soc 1991;89:371-476.

31 Augsburger JJ, Golden MI, Shields JA. Fluorescein angiography of choroidal malignant melanomas with retinal invasion. Retina 1984;4:232-41.

32 Daicker B. Retinal and papillary melanosis caused by a malignant choroidal melanoma bursting the retina into the vitreous body. Ophthalmologica 1973;166:460-71.

33 Leys AM, Van Eyck LM, Nuttin BJ, et al. Metastatic carcinoma to the retina. Clinicopathologic findings in two cases. Arch Ophthalmol 1990;108:1448-52.
34 Shields JA, Joffe L, Guibor P. Choroidal melanoma clinically simulating a retinal angioma. Am f Ophthalmol 1978;85: 7-71

35 Bardenstein DS, Char DH, Irvine AR, et al. Extramacular disciform lesions simulating uveal tumors. Ophthalmology 1992;99:944-51

36 Felder KS, Brockhurst RJ. Retinal neovascularization complicating rhegmatogenous retinal detachment of long duration. Am f Ophthalmol 1982;93:773-6.

37 Kreusel KM, Bornfeld N, Lommatzsch A, et al. Ruthenium106 brachytherapy for peripheral retinal capillary hemangioma. Ophthalmology 1998;105:1386-92.

38 Madreperla SA, Hungerford JL, Plowman PN, et al. Choroidal hemangiomas: visual and anatomic results of treatment by photocoagulation or radiation therapy. Ophthalmology 1997;104:1773-8.

39 Shields CL, Shields JA, Gunduz K, et al. Radiation therapy for uveal malignant melanoma. Ophthalmic Surg Lasers 1998;29:397-409. 